\title{
STRONGLY KOSZUL ALGEBRAS
}

\author{
JÜRGEN HERZOG, TAKAYUKI HIBI and GAETANA RESTUCCIA
}

\section{Introduction}

In this paper we introduce a condition for homogeneous $K$-algebras, which implies that these algebras are Koszul, that is, that their residue class field has a linear resolution.

Important classes of Koszul algebras, discovered by Fröberg [5], are the $K$-algebras $K\left[x_{1}, \ldots, x_{n}\right] / I$, where the generators of $I$ are quadratic monomials. As already observed in [4], any colon ideal of the form $\left(x_{i_{1}}, \ldots, x_{i_{k}}\right): x_{i_{K+1}}$ in a Fröberg ring is generated by a subsequence of $x_{1}, \ldots, x_{n}$. This observation, as indicated in [4], yields a simple proof of the fact that Fröberg rings are Koszul.

In this paper we call a homogeneous $K$-algebra $R$ strongly Koszul, if the graded maximal ideal of $R$ admits a minimal system of generators $\mathrm{x}=x_{1}, \ldots, x_{n}$ such that for each subsequence of the generators, a colon ideal as above, is again generated by a subsequence of $\mathbf{x}$. In Section 1 we prove some basic properties of strongly Koszul algebras, and show in particularr, that any ideal generated by a partial sequence of $\mathbf{x}$ has a linear resolution. Analyzing the proof one finds a very explicit formula for the Poincaré series of an ideal generated by a subsequence of $\mathbf{x}$.

The notion of strongly Koszul algebras is particularly interesting for a homogeneous semigroup $K[S]$. To each element $s \in S$ there is associated its divisor poset. It has been shown by Laudal and Sletsjøe [8] that the simplicial homology of the associated order complex is isomorphic to the sth graded component of $\operatorname{Tor}^{K[S]}(K, K)$. From this fact one deduces easily that $K[S]$ is Koszul if and only if all divisor posets are Cohen-Macaulay with respect to $K$; see for example [11] or [9]. On the other hand, the strongly Koszul property of $K[S]$ (with respect to the system of semigroup generators) is characterized by fact that all divisor posets of $S$ are wonderful, see 1.4. In the same proposition it is also shown that $S$ is strongly Koszul if and only if the intersection of any two principal ideals $\left(s_{1}\right) \cap\left(s_{2}\right)$ of generators of $S$ is gen-

Received July 15, 1997. 
erated in degree 2. We dot not know whether there is a natural bound for the degree of the generators of such intersections for arbitrary homogeneous semigroup rings. Denoting this bound by $b(S)$, it is clear that $b(S)=2$ if and ony if $S$ is strongly Koszul. In general one has, as noted in 1.7, that $b(S)$ is greater than or equal to the largest degree of a generating relation of the semigroup $S$. There is some computational evidence that $b(S) \leq 3$, if $S$ has a quadratic Gröbner basis.

In Section 2 we show that Veronese subrings, tensor and Segre products of strongly Koszul semigroup rings are again strongly Koszul. Starting with polynomial rings and applying these operations in any order we arrive at semigroups which are strongly Koszul. We call the semigroups resulting from these operations "elementary". The non-elementary strongly Koszul algebras seem to be very rare. One such example is given in 1.6 (3). Due to their rarity a classification of the strongly Koszul algebras seems not to be impossible, and indeed, for restricted classes can be done.

In the following Sections 3 and 4 we prove that among the Hibi rings (these are $K$-algebras defined by lattices; see [6] and [7]), and among the $K$ algebras defined by bipartite graphs, the only strongly Koszul algebras are those which arise from polynomial rings by repeated tensor and Segre product operations.

The authors would like to thank E. De Negri and V. Welker for several discussions in connection with this paper.

\section{Definition and basic properties of strongly Koszul algebras}

Let $K$ be a field, and let $R$ be a homogeneous $K$-algebra, that is, a finitely generated graded $K$-algebra which is generated over $K$ by elements of degree 1. We denote by $\mathbf{m}$ the graded maximal of $R$.

Definition 1.1. The homogeneous $K$-algebra $R$ is called strongly Koszul if its graded maximal ideal $m$ admits a minimal system of homogeneous generators $u_{1}, \ldots, u_{n}$ such that for all subsequences $u_{i_{1}}, \ldots, u_{i_{r}}$ of $u_{1},, u_{n}$ with $i_{1}<i_{2}<\ldots<i_{r}$, and for all $j=1, \ldots, r-1$, the colon ideal $\left(u_{i_{1}}, \ldots, u_{i_{j-1}}\right): u_{j}$ is generated by a subset of elements of $\left\{u_{1}, \ldots, u_{n}\right\}$.

The principal idea for the proof of the next theorem, which justifies our definition, can already been found in [4], where similar arguments are used in a more special situation.

THEOREM 1.2. Let $R$ be strongly Koszul with respect to the minimal homogeneous system $u_{1} \ldots, u_{n}$ of generators of the graded maximal ideal $\mathbf{m}$ of $R$. Then any ideal of the form $\left(u_{i_{1}}, \ldots, u_{i_{r}}\right)$ has a linear resolution. In particular, $R$ is Koszul. 
Proof. Just as in [4] we call a graded $R$-module $M$ linear if it admits a system of generators $g_{1}, \ldots, g_{m}$, all of the same degree, such that for $j=1, \ldots, m$ the colon ideals

$$
\left(R g_{1}+\ldots+R g_{j-1}\right): g_{j}=\left\{a \in R \mid a g_{j} \in\left(R g_{1}+\ldots+R g_{j-1}\right)\right\}
$$

are generated by subsets of $\left\{u_{1}, \ldots, u_{n}\right\}$.

Notice that all the ideals $\left(u_{i_{1}}, \ldots, u_{i_{r}}\right)$ are linear modules. Hence the theorem will be proved if we have shown that a linear module $M$ has linear relations, and that the syzygy module of a linear module is again linear.

The first property is immediately clear. Indeed, if $a_{1} g_{1}+\ldots+a_{m} g_{m}$ is a homogeneous generating relation of $M$, and $a_{j}$ is the last non-zero coefficient of this relation, then $a_{j}$ is a generator of the colon ideal $\left(R g_{1}+\ldots+R g_{j-1}\right): g_{j}$, and hence is of degree 1. Therefore, the relation is linear.

Let $\Omega^{1}(M)$ denote the first syzygy module of $M$. We prove by induction on the number of generators of $M$, that $\Omega^{1}(M)$ is a linear module. If $M$ is cyclic, then $\Omega^{1}(M)$ is an ideal generated by a subset of $\left\{u_{1}, \ldots, u_{n}\right\}$, and hence is linear. Now suppose that $M$ is generated by the $m$ elements $g_{1}, \ldots, g_{m}, m>1$, for which $M$ is linear. Then $N=R g_{1}+\ldots R g_{m-1}$ is also linear, and by induction hypothesis has a linear syzygy module $\Omega^{1}(N)$. Say, $\Omega^{1}(N)$ is linear with respect to its generators $h_{1}, \ldots, h_{k}$. Now we build a suitable system of generators of $\Omega^{1}(M)$ using the exact sequence

$$
0 \rightarrow \Omega^{1}(N) \rightarrow \Omega^{1}(M) \rightarrow \Omega^{1}(M / N) \rightarrow 0 .
$$

The module $M / N$ is cyclic with annihilator $\left(R g_{1}+\ldots+R g_{m-1}\right): g_{m}$, and so there exist $1 \leq i_{1}<i_{2}<\ldots<i_{l} \leq n$ such that $\Omega^{1}(M / N) \simeq\left(u_{i_{1}} \ldots, u_{i_{l}}\right)$. Now we choose homogeneous elements $h_{k+1}, \ldots, h_{k+l}$ in $\Omega^{1}(M)$ mapping onto $u_{i_{1}}, \ldots, u_{i_{i}}$. We leave it to the reader to check that $\Omega^{1}(M)$ is linear with respect to the generators $h_{1}, \ldots, h_{k+l}$.

One remarkable feature of Koszul algebras is that their Poincare series is a rational function. Indeed, one has the following well-known identity

$$
P_{K}(t) \cdot H_{R}(-t)=1
$$

for any Koszul algebra $R$. Here $P_{M}(t)=\sum_{i \geq 0} \operatorname{dim}_{K} \operatorname{Tor}_{i}^{R}(K, M) t^{i}$ denotes the Poincaré series, and $H_{M}(t)=\sum_{i>0} \operatorname{dim}_{K} M_{i} t^{i}$ the Hilbert series of a graded $R$-module $M$. One proves the identity, noting that the Hilbert series of $K$ is 1 , and that it can be computed uing the linear free $R$-resolution of $K$. The same argument shows that if $R$ is strongly Koszul, and $I=\left(u_{i_{1}}, \ldots, u_{i_{k}}\right)$, then

$$
P_{R / I}(t) \cdot H_{R}(-t)=H_{R / I}(-t) .
$$


The proof of Theorem 1.2 gives a more precise information about the Poincaré series. For any two subsets $U, V \subset[n]=\{1, \ldots, n\}$, say $U=\left\{i_{1}, \ldots, i_{k}\right\}$ with $i_{1}<i_{2}<\ldots<i_{k}$, we denote by $a_{U, V}$ the number of ideals $I(V)=\left(u_{i}\right)_{i \in V}$ which are of the form $\left(u_{i_{1}}, \ldots, u_{i_{j-1}}\right) ; U_{i_{j}}$ for some $j=1, \ldots, k$. Now we choose some linear order of the subsets of $[n]$, in other words a bijective map $i: B_{[n]} \rightarrow\left\{1, \ldots, 2^{n}\right\}$, where $B_{[n]}$ denotes the set of all subset of $[n]$, and consider the integer matrix $A=\left(a_{U, V}\right)$ where the entry $a_{U, V}$ is at position $(i(V), i(U))$.

The arguments in 1.2 then yield that

$$
P_{I(U)}(t)=|U|+\sum_{V} a_{U, V} P_{I(V)}(t) t,
$$

where $|U|$ denotes the number of elements of $U$. Applying this formula recursively, we obtain the following formal power series with integer coefficients:

$$
P_{I(U)}(t)=\sum_{i \geq 0} e_{U} A^{i} w t^{i}
$$

where $e_{u}$ is the row vector whose entries are all zero, except the $i(U)$-th entry, which is 1 , and where $w$ is the column vector whose $i(V)$-th entry is $|V|$ for all $V \subset[n]$. We set

$$
B=\sum_{i \geq 0} A^{i} t^{i}
$$

Then $B$ is a matrix with coefficients in $Z[[t]]$, and we have

$$
B=(E-A t)^{-1} \text { and } P(t)=B w,
$$

where $P(t)$ is the column vector with $i(U)$-th entry $P_{I(U)}$ for all $U \subset[n]$. It follows that $w=(E-A t) P(t)$. Thus, if we set $C=E-A t$, and denote by $C_{U, V}$ the submatrix of $C$ which is obtained from $C$ by deleting the $i(U)$-th row and the $i(V)$-th column, Cramer's rule implies

COROLlary 1.3. Let $R$ be strongly Koszul. Then with the notation introduced one has

$$
\left.P_{I(U)}=\left(\sum_{V}(-1)^{i(U)+i(V)}|V| \operatorname{det} C_{U, V}\right) \operatorname{det} C\right)^{-1} .
$$

Natural classes of $K$-algebras to which our theory may be applied are the semigroup rings of homogeneous semigroups because their graded maximal ideal has a distinguished basis. We call a finitely generated subsemigroup $S$ of $\mathrm{N}^{n}$ homogeneous if $S$ can be written as a disjoint union 


$$
S=\bigcup_{i \geq 0} S_{i}
$$

with $S_{0}=\{0\}, S_{i}+S_{j} \subset S_{i+j}$ for all $i, j$, and $S$ generated by the elements of $S_{1}$. The elements of $S_{i}$ are called elements of degree $i$.

It is clear that the semigroup ring $R=K[S]$ of a homogeneous semigroup is a homogeneous $K$-algebra, with homogeneous components $R_{i}=K S_{i}$. We identify $R$ with the subring of the polynomial ring $K\left[x_{1}, \ldots, x_{n}\right]$ with $K$-basis $x^{a}=x_{1}^{a_{1}} \ldots x_{n}^{a_{n}}, a \in S$.

Typical examples of homogeneous semigroup rings are the $K$-subalgebras of a polynomial ring which are generated by a set of monomials which are all of same degree.

Let $S$ be a homogeneous semigroup, and let $a_{1}, \ldots, a_{m}$ be its degree one generators. Then $R=K[S]$ is generated by the monomials $u_{i}=x^{a_{i}}$ for $i=1, \ldots, m$. We say that $S$ is strongly Koszul, if $R$ is strongly Koszul with respect to the generators $u_{1} \ldots, u_{m}$. Obviously, this property only depends on $S$, and not on the field $K$.

It is convenient to identify the elements of the semigroup $S$ with the corresponding monomials in $K[S]$. In particular the $u_{i}$ will be identified with the generators of $S$.

The next simple proposition interprets the property "strongly Koszul" in terms of the divisor poset of $S$. We consider $S$ a poset, called the divisor poset of $s$, by introducing the following partial order: let $s_{1}, s_{2} \in S$; then

$s_{1} \leq s_{2}, \quad$ if there exists $t \in S$ such that $s_{2}=s_{1}+t$.

Notice that $S$ is a locally finite poset, in other words, any interval $[s, t]=\{a \in S \mid s \leq a \leq t\}$ is finite. Let $\Pi$ be an arbitrary poset, and $u, v \in \Pi$. One says that $u$ covers $v$, if $v<u$ and there is no $w$ with $v<w<u$.

The locally finite poset is called wonderful or locally upper semimodular if whenever $v_{1}$ and $v_{2}$ cover $u$ and $v_{1}, v_{2}<v$ for some $v \in \Pi$, then there exists $t \in \Pi, t \leq v$, which covers each of $v_{1}$ and $v_{2}$.

Proposition 1.4. Let $S$ be a homogeneous semigroup, and let $u_{1}, \ldots, u_{m} \in$ $K[S]$ be the generators of $S$. Then the following conditions are equivalent:

(a) $S$ is strongly Koszul;

(b) the divisor poset of $S$ is wonderful;

(c) the ideals $\left(u_{i}\right) \cap\left(u_{j}\right)$ are generated in degree 2 for all $i \neq j$.

Proof. The equivalence of (b) and (c) is straightforward. In order to prove that (c) implies (a) we notice that there is a graded isomorphism

$$
\left(u_{i}\right):\left(u_{j}\right) \rightarrow\left(\left(u_{i}\right) \cap\left(u_{j}\right)\right)(-1)
$$

which maps an element $a \in\left(u_{i}\right):\left(u_{j}\right)$ to $a u_{j} \in\left(u_{i}\right) \cap\left(u_{j}\right)$. Since $S$ is strongly 
Koszul, the ideal $\left(u_{i}\right):\left(u_{j}\right)$ is generated in degree 1 , and so $\left(u_{i}\right) \cap\left(u_{j}\right)$ is generated in degree 2 .

Conversely, suppose that condition (c) is satisfied, and let $a \in\left(u_{i-1}, \ldots, u_{j-1}\right): u_{i_{j}}$. We use the fact that $K[S]$ has a multi-graded structure (where the monomials is given the multi-degree determined by their exponent). Thus, since the $u_{i}$ are multi-homogeneous, it follows that the colon ideal $\left(u_{i_{1}}, \ldots, u_{i_{j-1}}\right): u_{i_{j}}$ is multi-graded, and hence is generated by monopmials. Therefore we may assume that $a$ is a monomial, and conclude that $a u_{i_{j}}=b u_{i_{k}}$ for some $k \leq j-1$ and some monomials $b$. But this implies that $a u_{i_{j}} \in\left(u_{i_{j}}\right) \cap\left(u_{i_{k}}\right)$. Now if we assume that $a$ is a generator of the colon ideal, then it follows that $a u_{i_{j}}$ is a generator of $\left(u_{i_{j}}\right) \cap\left(u_{i_{k}}\right)$. By assumption, this ideal is generated in degree 2 , so that $a$ is of degree 1 , as desired.

Corollary 1.5. Let $S$ be a strongly Koszul semigroup, $u$ and $v$ be two semigroup elements of degree $n$ and $m$, respectively. Then the ideal $(u) \cap(v)$ is generated in degree $\leq n+m$.

Proof. We proceed by induction on degree $n+m$. For $n+m=2$, the assertion follows from 1.4. Now let $n+m>2$. Then we may assume that $n>1$, and we write $u=u^{\prime} u_{i}$ where $u^{\prime}$ is of degree $n-1$ and $u_{i}$ is a semigroup generator. Now let $w \in(u) \cap(w)$. Then $w \in\left(u^{\prime}\right) \cap(v) \cap\left(u_{i}\right)$. Therefore there exists a generator $z \in\left(u^{\prime}\right) \cap(v)$ such that $w \in(z) \cap\left(u_{i}\right)$. Our induction hypothesis implies that $\operatorname{deg} z \leq n+m-1$. Let $z^{\prime}=z / u^{\prime}$ and $w^{\prime}=w / u^{\prime}$. Then $w^{\prime} \in\left(z^{\prime}\right) \cap\left(u_{i}\right)$. Let $y$ be a generator of $\left(z^{\prime}\right) \cap\left(u_{i}\right)$ such that $w^{\prime} \in\left(y^{\prime}\right)$. since $\operatorname{deg} z^{\prime}+\operatorname{deg} u_{i} \leq 1+m<n+m$, we have $\operatorname{deg} y^{\prime} \leq 1+m$, by induction hypothesis. Set $y=y^{\prime} u^{\prime}$; then $y \in(u) \cap(v), \operatorname{deg} y \leq n+m$ and $w \in(y)$, as desired.

Let $R$ be the semigroup ring of a homogeneous semigroup $S$. Then one has the following hierarchy of properties:

The defining of $R$ has a Gröbner basis of quadrics, or $R$ is strongly Koszul $\Rightarrow R$ is Koszul $\Rightarrow R$ is defined by quadrics.

We do not know whether the defining ideal of a stongly Koszul semigroup has a Gröbner basis of quadrics.

In concluding this section we consider a few

EXAMPLeS 1.6. (1) The semigroup ring

$$
R=K\left[x_{1} s, \ldots, x_{n} s, x_{1} t, \ldots, x_{n} t\right]
$$

is strongly Koszul. Indeed, $R$ has the $K$-basis $x^{a} s^{i} t^{j}$ with $a \in \mathrm{N}^{n}$, and $|a|=i+j$ where $|a|=\sum_{k=1}^{n} a_{k}$. Suppose $u \in\left(x_{k} s\right) \cap\left(x_{l} s\right), k \neq l, u=x^{a} s^{i} t^{j}$. Then $i \geq 1$; if $i=1$, then $j \geq 1$, and $x_{k} x_{l} s t$ divides $u$, and if $i>1$, then $x_{k} x_{l} s^{2}$ 
divides $u$. In any case we see that $u$ is a multiple of an element of degree 2 of the intersection $\left(x_{k} s\right) \cap\left(x_{l} s\right)$.

We also have to consider the case that $u \in\left(x_{k} s\right) \cap\left(x_{l} t\right)$. It is clear that $i \geq 1$ and $j \geq 1$. If $k \neq l$, then $x_{k} x_{l}$ st divides $u$, and if $k=l$ either $a_{k}>1$, in which case $x_{k}^{2} s t$ divides $u$, or $a_{r} \geq 1$ for some other $r$. In this case $x_{k} x_{r} s t$ divides $u$. Again we see that in all cases $u$ is a multiple of an element of degree 2 in the intersection $\left(x_{k} s\right) \cap\left(x_{l} t\right)$.

This example is part of a more general class of strongly Koszul algebras studied in the next section.

(2) Let $S$ be a homogeneous semigroup which is defined by one quadric: In other words, $K[S] \simeq K\left[x_{1}, \ldots, x_{n}\right] /(f)$ where $f=v-w$ is a quadratic binomial. We claim that $S$ is strongly Koszul. In order to prove this we show that for any two numbers $1 \leq i<j \leq n$, the annihilator of $u_{j}$ in $K[S] /\left(u_{i}\right)$ is generated in degree 1 . Here, as before, the $u_{i}$ denote the generators of $S$, that is, the residues of the $x_{i}$ modulo $f$.

If $x_{i}$ does not divide $v$ or $w$, then $u_{j}$ is regular on $K[S] /\left(u_{i}\right)$. In this case the assertion is trivial. On the other hand, if $x_{i}$ divides, say $v$, and $w=x_{k} x_{l}$, then $u_{j}$ is again regular on $K[S]$ in case $j \neq k$ and $j \neq l$, while the annihilator of $u_{j}$ is $\left(u_{l}\right)$ in case $j=k$, and is $\left(u_{k}\right)$ in case $j=l$

(3) We denote by $R_{n, d}$ the $K$-subalgebra of the polynomial ring in $n$ variables which is generated by all squarefree monomials of degree $d$. It has been shown by Sturmsfels [12] that the defining ideals of these algebras (which we call squarefree Veronese rings) have a quadratic Gröbner basis, and thus these algebras are Koszul. We refer the reader to the paper De Negri [10] for related results.

We claim that for $n \geq 2 d+[d / 2], R_{n, d}$ is not strongly Koszul. Here we denote by $[x]$ the integer part of a real number $x$. We prove the assertion for $d$ even (the proof for $d$ odd is similar). We consider $R_{n, d}$ as the subring of the polynomial ring $S$ in the variables $x_{1}, \ldots, x_{d}, y_{1}, \ldots, y_{d}, z_{1} \ldots, z_{[d / 2]}, \ldots \mathrm{We}$ first notice that the $i$-th graded component of $R_{n, d}$ has a $K$-basis consisting of all monomials of degree $i d$ whose exponents are bounded by $i$. Therefore we see that

$$
u=\left(x_{1} \ldots x_{d}\right)\left(y_{1} \ldots y_{d}\right)\left(z_{1}^{2} \ldots z_{d / 2}^{2}\right)
$$

belongs to $R_{n, d}$. Set $u_{1}=x_{1} \ldots x_{d}$ and $u_{2}=y_{1} \ldots y_{d}$; then it is obvious that the only generator of degree 2 of $\left(u_{1}\right) \cap\left(u_{2}\right)$ is $u_{1} u_{2}$. Note that $u$ is not a multiple of $u_{1} u_{2}$ since the complementary factor $z_{1}^{2} \ldots z_{d / 2}^{2}$ does not belong to $R_{n, d}$. On the other hand $u \in\left(u_{1}\right) \cap\left(u_{2}\right)$, so that $u$ is a generator of degree 3 of $\left(u_{1}\right) \cap\left(u_{2}\right)$.

For $d=2$ the bound for $n$ is the best possible. Indeed, one easily checks that $R_{4,2}$ is strongly Koszul. 
REMARK 1.7. In general it seems hard to determine the degree of the generators of the intersections $\left(u_{i}\right) \cap\left(u_{j}\right)$ for a homogeneous semigroup ring $K[S]=K\left[u_{1}, \ldots, u_{n}\right]$. We denote by $b(S)$ the highest possible degree of a generator of any such intersection.

Let $I$ be the defining ideal of $K[S]$. The ideal $I$ is generated by binomials. Let $d$ be the highest degree of an element in a minimal set $\mathscr{M}$ of binomial generators of $I$. Then one has $b(S) \geq d$. Indeed, let $\epsilon: K\left[x_{1}, \ldots, x_{n}\right] \rightarrow$ $K\left[u_{1}, \ldots, u_{n}\right]$ be the canonical epimorphism with $\epsilon\left(x_{i}\right)=u_{i}$ for $i=1, \ldots, n$, and suppose that $f=f_{1}-f_{2}$ is a binomial of $\mathscr{M}$. Suppose further that $x_{i} \mid f_{1}$ and $x_{j} \mid f_{2}$. Then, $a=u_{i} u=u_{j} v$, where $a=\epsilon\left(f_{1}\right), u=\epsilon\left(f_{1} / x_{i}\right)$ and $v=\epsilon\left(f_{2} / x_{j}\right)$. Therefore, $a \in\left(u_{i}\right) \cap\left(u_{j}\right)$.

We claim that $a$ is a generator of $\left(u_{i}\right) \cap\left(u_{j}\right)$. Of course, the desired inequality will follows then from this assertion.

Suppose $a$ is not a generator of $\left(u_{i}\right) \cap\left(u_{j}\right)$. Then there exists a monomial (of the generators $\left.u_{1}, \ldots, u_{n}\right) a^{\prime} \in\left(u_{i}\right) \cap\left(u_{j}\right)$ with $a=a^{\prime} d$ for some monomial $d$. Say, $a^{\prime}=u_{i} u^{\prime}=u_{j} v^{\prime}$. Then

$$
0=u_{i} u-u_{j} v=d\left(u_{i} u^{\prime}-u_{j} v^{\prime}\right)+u_{i}\left(u-d u^{\prime}\right)-u_{j}\left(v-d v^{\prime}\right) .
$$

The binomials on the right hand side are all equal to zero, so they correspond to binomial relations. Thus this equations tells us that $f$ is not a minimal binomial relation, a contradiction.

\section{Classes of strongly Koszul algebras}

In this section we consider certain ring constructions which yield classes of strongly Koszul algebras.

Proposition 2.1. Let $S$ be a strongly Koszul semigroup, and let $u_{1}, \ldots, u_{m} \in K[S]$ be the generators of the graded maximal ideal of $R=K[S]$ which correspond to the generators of $S$. Then

(a) $R /\left(u_{i_{1}}, \ldots, u_{i_{k}}\right)$ is strongly Koszul for any subsequence $u_{i_{1}}, \ldots, u_{i_{k}}$ of the generators $u_{1}, \ldots, u_{n}$.

(b) $R / J$ is strongly Koszul for any ideal $J \subset R$ generated by monomials of degree 2 in the $u_{i}$ such that for all monomials $u_{i} u_{j}$ in $J$ and all $k \neq i, j$ one has $\left(u_{i} u_{j}\right): u_{k}+J=\left(u_{i}: u_{k}\right)=\left(u_{j}: u_{k}\right)+J$.

Proof. Let $L$ be any ideal in $R$ generated by monomials in the $u_{i}$. Then $R / L$ is multigraded. Therefore if we denote by $\bar{u}_{i}$ the residue class of $u_{i}$ in $R / L$, then the strongly Koszul property for $R / L$ is equivalent to the following two conditions:

(1) for all $k$ with $\bar{u}_{k} \neq 0$, the annihilator of $\bar{u}_{k}$ is generated in degree 1 , and

(2) $\left(\bar{u}_{i}\right) \cap\left(\bar{u}_{j}\right)$ is generated in degree 2 for all $i \neq j$. 
We leave it to the reader to check (1) and (2) in case (a). Now suppose we are in case (b), and let $\bar{v} \neq 0$ be a monomial in $R / J$ annihilating some $\bar{u}_{k} \neq 0$. Then $v u_{k} \in J$, and so $v \in\left(u_{i} u_{j}\right): u_{k}$ for some $u_{i} u_{j} \in J$. If $k \neq i, j$, then our hypotheses imply that $v \in\left(u_{i}: u_{k}\right)$ or $v \in\left(u_{j}: u_{k}\right)$. Thus, since $R$ is strongly Koszul, it follows that $v=w u_{l}$ for which $u_{l} u_{k} \in\left(u_{i} u_{j}\right)$. If $k=i$ or $k=j, v$ is a multiple of $u_{i}$ or of $u_{j}$. In both cases one concludes that the annihilator of $\bar{u}_{k}$ is generated in degree 1. Condition (2) for the ring $R / J$ is inherited from $R$.

As consequence of 2.1 (b) we have that Fröberg rings are strongly Koszul, a result which is already implicitly contained in [4].

COROLLARY 2.2. Let $R$ be the factor ring of a polynomial ring by quadratic monomials. Then $R$ is strongly Koszul.

Other classes of examples are derived from the tensor product. Let $R$ and $P$ be homogeneous $K$-algebras. The tensor product $R \otimes_{K} P$ is again a homogeneous $K$-algebra with homogeneous components $(R \otimes P)_{i}=\bigoplus_{j+k=i}$ $R_{j} \otimes_{K} R_{k}$. The Segre product $R * P$ of $R$ and $P$ is the homogeneous subalgebra of $R \otimes_{k} P$ with homogeneous components $(R * P)_{i}=R_{i} \otimes_{k} P_{i}$, while the fibre product $R \circ P$ of $R$ and $P$ is defined to be the factor ring of $R \otimes_{K} P$ modulo the ideal generated by all elements $r \otimes s$ with $r \in R_{1}$ and $s \in P_{1}$. Finally, the $d$-the Veronese ring of $R$ is the subring $R^{(d)}$ of $R$ with homogeneous components $\left(R^{(d)}\right)_{i}=R_{i d}$.

Now we can show the following result, whose analogue is partly known for Koszul algebras by Backelin and Fröberg [2].

Proposition 2.3. Let $S$ and $T$ be homogeneous semigroups, $R=K[S]$ and $P=K[T]$ the corresponding semigroup rings over some field $K$, and $Q$ be the tensor product, the Segre product, or the fiber product of $R$ and $P$. Then

(a) the Veronese rings $R^{(d)}$ are strongly Koszul if $R$ is strongly Koszul;

(b) $Q$ is strongly Koszul, if and only if $R$ and P are strongly Koszul.

Proof. (a) In order to show that the Veronese rings $R^{(d)}$ are strongly Koszul, we note that $R^{(d)}=K\left[S^{(d)}\right]$ where $S^{(d)}$ is the homogeneous subsemigroup $\bigcup_{i} S_{i d}$ of $S$. Notice further that $(S, \leq)$ is a graded poset since $S$ is homogeneous. Hence it is clear that our assertion follows once we have proved the following statement: suppose that $S$ is a graded, wonderful poset. Given elements $u, v_{1}, v_{2} \in S$ with $u<v_{1}, v_{2}<v$ and such that $\operatorname{deg}\left(v_{1}\right)-\operatorname{deg}(u)=i$ and $\operatorname{deg}\left(v_{2}\right)-\operatorname{deg}(u)=j$. Then there exists $t \leq v$ with $\operatorname{deg}(t)-\operatorname{deg}(u)=i+j \operatorname{such}$ that $v_{1}, v_{2}<t$.

This simple and well-known combinatorial statement is proved by induction on $i+j$. Subtracting from all elements the smallest element $u$, we may assume that $u=0$. If $i+j=2$, then this is just the property wonderful. Now 
supppose that $i+j>2$, and, say, $i \geq 2$. Then there is an element $w$ of degree $i-1$ with $0<w<v_{1}$. By induction hypothesis, there exists an element $s \in S$ of degree $i+j-1$ with $w, v_{2}<s \leq v$ Now we apply again our induction hypothesis to the situation $w<v_{1}, s \leq v$, and find an element $t \leq v$ of degree $\operatorname{deg}\left(v_{1}\right)+\operatorname{deg}(s)-2 \operatorname{deg}(w)=i+j$ with $v_{1}, s<t$. This proves the assertion.

(b) Observe that $R \otimes P \simeq K[S \times T]$ where $S \times T$ is the product of the two semigroups $S$ and $T$. The elements of $S \times T$ are the pairs $(s, t)$ with $s \in S$ and $t \in T$, and with componentwise addition. We leave it to the reader to check the easy fact that $(S \times T, \leq)$ is wonderful if $(S, \leq)$ and $(T, \leq)$ are wonderful.

To show that the Segre product $R * P$ is strongly Koszul is even simpler. We have $R * P=K[S * T]$ where $S * T$ is the subsemigroup of $S \times T$ consisting of all elements $(s, t)$ with $\operatorname{deg}(s)=\operatorname{deg}(t)$. Now suppose $(s, t)$ and $(u, v)$ cover $(a, b)$ and $(s, t),(u, v)<(f, g)$. Then $s$ and $u$ cover $a, t$ and $v$ cover $b, u<f$ and $t, v<g$. Therefore, there exist $k \in S$ and $l \in T$ such that $k$ covers $s$ and $u, l$ covers $t$ and $v$, and such that $k \leq f$ and $l \leq f$. Then $(k, l)$ covers $(s, t)$ and $(u, v)$ and $(k, l) \leq(f, g)$.

The assertion for the fiber product follows from 2.1 (b).

Conversely assume that $Q$ is strongly Koszul (with respect to the natural algebra generators, say, $\left.w_{1}, \ldots, w_{k}\right)$. Let $R=K\left[u_{1}, \ldots, u_{n}\right]$ and $P=$ $K\left[v_{1}, \ldots, v_{m}\right]$ where the generators $u_{i}$ and $v_{j}$ correspond the generators $S$ and $T$. We will see that there exist subsequences $W_{1}$ and $W_{2}$ of $w_{1}, \ldots, w_{k}$ such that $Q /\left(W_{1}\right) \simeq R$ and $Q /\left(W_{2}\right) \simeq P$. Hence for the tensor product and the Segre product it follows from 2.1 (a) that $R$ and $S$ are strongly Koszul. In the proof of 2.1 (a) we made use of the fact that a semigroup ring is multigraded. Since the fibre product of two semigroup rings is multi-graded too, the proof given there carries over, so that 2.1 (a) is also valid for the fiber product. Thus if the fiber product is strongly Koszul, we also conclude that $R$ and $S$ are strongly Koszul.

The choice of the sets $W_{1}$ and $W_{2}$ is as follows: if $Q$ is the tensor or fiber product, we let $W_{1}=\left\{1 \otimes v_{1}, \ldots, 1 \otimes v_{m}\right\}$ and $W_{2}=\left\{u_{1} \otimes 1, \ldots, u_{n} \otimes 1\right\}$; if $Q$ is the Segre product, we let $W_{1}=\left\{u_{i} \otimes v_{j} \mid i=2, \ldots, n, j=1, \ldots, m\right\}$ and $W_{2}=\left\{u_{i} \otimes v_{j} \mid i=1, \ldots, n, j=2, \ldots, m\right\}$.

We dot not know whether 2.3 holds more generally for arbitrary strongly Koszul algebras as defined in 1.1.

It is known [1] that the sufficiently high Veronese subrings of an arbitrary homogeneous $K$-algebra are Koszul. The following example shows that this is not true for the property "strongly Koszul". Indeed, consider in $K\left[x_{1}, x_{2}, x_{3}\right]$ the lexsegment $L$ in degree 2 with smallest element $x_{2} x_{3}$, that is, the set of monomials 


$$
\left\{x_{1}^{2}, x_{1} x_{2}, x_{2} x_{3}, x_{2}^{2}, x_{2} x_{3}\right\},
$$

and let $R$ be the homogeneous $K$-algebra generated over $K$ by these monomials. For each $d$, the element $\left(x_{1}^{2 d}\right)\left(x_{2}^{d} x_{3}^{d}\right)^{2}$ of degree 3 in $R^{(d)}$ is a generator of the intersection $\left(x_{1}^{2 d}\right) \cap\left(x_{2}^{2 d}\right)$. In other words, no Veronese subring of $R$ is strongly Koszul.

Definition 2.4. We call a semigroup $S$ trivial if, starting with polynomial rings, $K[S]$ is obtained by repeated applications of Segre products and tensor products.

In the next sections we will show that for certain classes of semigroup rings, the only strongly Koszul algebras are the trivial ones. On the other hand, the ring $R_{2,4}$ in 1.6 (2) is an example of a non-trivial Strongly Koszul algebra.

\section{The classification of strongly Koszul Hibi rings}

The Segre product of two polynomial rings may be viewed as the Hibi ring of the product poset of two chains, and hence such a ring is strongly Koszul. In this section we classify all distributive lattices whose Hibi rings are strongly Koszul.

Let $L$ be a finite distributive lattice and $K\left[x_{\alpha} \mid \alpha \in L\right]$ the polynomial ring in $|L|$ indeterminates over a field $K$. Following [6] (see also [7]) we define the Hibi ring $\mathscr{R}_{K}[L]$ associated with the lattice $L$ by

$$
\mathscr{R}_{K}[L]=K\left[x_{\alpha} \mid \alpha \in L\right] /\left(x_{\alpha} x_{\beta}-x_{\alpha \wedge \beta} x_{\alpha \vee \beta} \mid \alpha, \beta \in L\right) .
$$

Then, $\mathscr{R}_{k}[L]$ is, in fact, a normal affine homogeneous semigroup ring. Moreover, $\mathscr{R}_{k}[L]$ is an algebra with straightening laws on $L$ over $K$. Let $\mathscr{B}(L ; d)$ denote the set of those monomials of $\mathscr{R}_{K}[L]$ of the form $x_{\alpha_{1}} x_{\alpha_{2}} \cdots x_{\alpha_{d}}$ with each $\alpha_{i} \in L$ such that $\alpha_{1} \leq \alpha_{2} \leq \cdots \leq \alpha_{d}$ in $L$ for $d=1,2 \ldots$, and set $\mathscr{B}(L ; 0)=\{1\}$. Then, $\bigcup_{d=0}^{\infty} \mathscr{B}(L ; d)$ is a $K$-basis of $\mathscr{R}_{K}[L]$. Thus, given any monomial $u=x_{\beta_{1}} x_{\beta_{2}} \cdots x_{\beta_{d}}$ of $\mathscr{R}_{K}[L]$ of degree $d$, there exists a unique monomial $v \in \mathscr{B}(L ; d)$ with $v=u$. We call such a monomial $v \in \mathscr{B}(L ; d)$ the standard expression of $u$. If $v=x_{\alpha_{1}} x_{\alpha_{2}} \cdots x_{\alpha_{d}}$ with $\alpha_{1} \leq \alpha_{2} \leq \cdots \leq \alpha_{d}$ is the standard expression of $u=x_{\beta_{1}} x_{\beta_{2}} \cdots x_{\beta_{d}}$, then it follows that

$$
\alpha_{k}=\bigwedge_{1 \leq i_{1}<i_{2}<\cdots<i_{k} \leq d}\left(\beta_{i_{1}} \vee \beta_{i_{2}} \vee \cdots \vee \beta_{i_{k}}\right)
$$

for each $1 \leq k \leq d$. 
The following theorem follows from our classification of strongly Koszul Hibi rings. However, its short and direct proof may be of interest.

Theorem 3.1. If L is Boolean, then $\mathscr{B}_{K}[L]$ is strongly Koszul.

PROOF. Fix arbitrary two monomial generators $x_{\alpha}$ and $x_{\beta}$ of $\mathscr{R}_{K}[L]$. Our goal is to prove that the ideal $\left(x_{\alpha}\right) \cap\left(x_{\beta}\right)$ is generated in degree 2 .

We first show that $x_{\xi} x_{\eta}$, where $\xi, \eta \in L$ with $\xi \leq \eta$, belongs to $\left(x_{\alpha}\right) \cap\left(x_{\beta}\right)$ if and only if $\xi \leq \alpha \wedge \beta$ and $\alpha \vee \beta \leq \eta$. In fact, if $x_{\xi} x_{\eta}=x_{\alpha} x_{\gamma}=x_{x} \delta$ for some $\gamma, \delta \in L$, then $\xi=\alpha \wedge \gamma=\beta \wedge \delta$ and $\eta \vee \gamma=\beta \vee \delta$. Thus, $\xi \leq \alpha \wedge \beta$ and $\alpha \vee \beta \leq \eta$. Now, suppose that $\xi \leq \alpha \wedge \beta$ and $\alpha \vee \beta \leq \eta$. Since $L$ is Boolean, the closed interval $[\xi, \eta]$ of $L$ is again Boolean. Thus, we can find $\gamma, \delta \in[\xi, \eta]$ such that $\alpha \wedge \gamma=\xi, \alpha \vee \gamma=\eta \quad$ and $\beta \wedge \delta=\xi, \beta \vee \delta=\eta$. Hence, $x_{\xi} x_{\eta}=x_{\alpha} x_{\gamma}=x_{\beta} x_{\delta}$. Thus, $x_{\xi} x_{\eta} \in\left(x_{\alpha}\right) \cap\left(x_{\beta}\right)$, as desired.

If $u=x_{\xi_{1}} x_{\xi_{2}} \cdots x_{\xi_{d}}$ with $\xi_{1} \leq \xi_{2} \leq \cdots \leq \xi_{d}$ belongs to $\left(x_{\alpha}\right) \cap\left(x_{\beta}\right)$ and if $u=x_{\alpha} x_{\gamma_{1}} \cdots x_{\gamma_{d-1}}=x_{\beta} x_{\delta_{1}} \cdots x_{\delta_{d-1}}$ for some $\gamma_{i}^{\prime}$ 's and $\delta_{j}$ 's in $L$, then the existence and uniqueness of standard expressions (1) guarantee that

$$
\begin{aligned}
& \xi_{1}=\alpha \wedge \gamma_{1} \wedge \cdots \wedge \gamma_{d-1}=\beta \wedge \delta_{1} \wedge \cdots \wedge \delta_{d-1} \\
& \xi_{d}=\alpha \vee \gamma_{1} \vee \cdots \vee \gamma_{d-1}=\beta \vee \delta_{1} \vee \cdots \vee \delta_{d-1}
\end{aligned}
$$

Thus, in particular, $\xi_{1} \leq \alpha \leq \xi_{d}$ and $\xi_{1} \leq \beta \leq \xi_{d}$, i.e., $\xi_{1} \leq \alpha \wedge \beta$ and $\alpha \vee \beta \leq \xi_{d}$. Hence, $\xi_{1} \xi_{d}$ belongs to $\left(x_{\alpha}\right) \cap\left(x_{\beta}\right)$. Thus, $\left(x_{\alpha}\right) \cap\left(x_{\beta}\right)$ is generated in degree 2 , as required.

Birkhoff's fundamental structure theorem [3] for finite distributive lattices guarantees that, for any finite distributive lattice $L$, there exists a unique poset $P$ such that $L$ is isomorphic to the lattice $J(P)$ consisting of all poset ideals of $P$, ordered by inclusion. Suppose $P$ contains an element $p$ wich is comparable with any other element of $P$. Consider the subposets $P_{1}=\{q \in P \mid q<p\}$ and $P_{2}=\{q \in P \mid q>p\}$ of $P$, and set $L_{1}=J\left(P_{1}\right)$, $L_{2}=J\left(P_{2}\right)$ and $L=J(P)$. then it is easy to see that $\mathscr{R}_{K}[L] \simeq$ $\mathscr{R}_{K}\left[L_{1}\right] \otimes \mathscr{R}_{K}\left[L_{2}\right]$. Thus in view of $2.3, \mathscr{R}_{K}[L]$ is strongly Koszul if and only if $\mathscr{R}_{K}\left[L_{1}\right]$ and $\mathscr{R}_{K}\left[L_{2}\right]$ are strongly Koszul.

The poset $P$ is simple if there is no element of $P$ which is comparable with any other element of $P$. Hence in order to find distributive lattices whose Hibi rings are strongly Koszul it suffices to consider lattices $J(P)$ of simple posets $P$. Another reduction is possible: one calls a poset connected, if its Hasse diagram is connected. Suppose the poset $P$ is not connected. Then there exist two non-empty subposets $P_{1}$ and $P_{2}$ of $P$ such that the elements of $P_{1}$ and $P_{2}$ are incomparable. Let $L_{1}=J\left(P_{1}\right), L_{2}=J\left(P_{2}\right)$ and $L=J(p)$; it is not difficult to see that $\mathscr{R}_{K}[L] \simeq \mathscr{R}_{K}\left[L_{1}\right] * \mathscr{R}_{K}\left[L_{2}\right]$. In particular, it follows 
from 2.3 that $\mathscr{R}_{K}[L]$ is strongly Koszul if and only if, for all connected components $L^{\prime}$ of $L, \mathscr{R}_{k}\left[L^{\prime}\right]$ are strongly Koszul.

Starting from polynomial rings, repeated application of Segre products and tensor products strongly produces Koszul Hibi rings. Such a Hibi ring is called trivial.

Now we are ready to formulate our classification result.

TheOREM 3.2. A Hibi ring is strongly Koszul if and only if it is trivial.

We observed already one implication. The other one will follow from

Theorem 3.3. Suppose that a finite poset $P$ is simple and connected. Then, the Hibi ring $\mathscr{R}_{K}[L]$ associated with the distributive lattice $L=J(P)$ is not strongly Koszul.

The proof of this theorem is a consequence of the next two lemmata.

Lemma 3.4. Suppose that a finite poset $P$ possesses a saturated chain $c_{1}<c_{2}<\cdots<c_{m}(m \geq 2)$ together with $a, b \in P$ such that (i) $c_{m}$ covers $b$; (ii) a covers $c_{1}$; (iii) $c_{1} \not \leq b$; (iv) $a \not \leq c_{m}$. Let $L=J(P)$ denote the finite distributive lattice consisting of all poset ideals of $P$. Then, $\mathscr{R}_{K}[L]$ is not strongly Koszul.

Proof. Let $I$ denote the poset ideal of $P$ consisting of those elements $q \in P$ with $c_{1} \not \leq q$ and $b \not \leq q$. Also, set $(b, a):=\{q \in P \mid b<q<a\}$ (possibly, empty) and $\left[c_{1}, c_{m}\right]:=\left\{q \in P \mid c_{1} \leq q \leq c_{m}\right\}$. We then define the elements $\zeta, \xi, \eta, \alpha$ and $\beta$ belonging to $L=J(P)$ by

$$
\begin{gathered}
\zeta=I, \xi=I \cup\left\{b, c_{1}\right\}, \eta=I \cup\{a, b\} \cup(b, a) \cup\left[c_{1}, c_{m}\right], \\
\alpha=I \cup\left\{c_{1}\right\}, \beta=I \cup\{a, b\} \cup(b, a\} \cup\left\{c_{1}\right\} .
\end{gathered}
$$

Then, in $\mathscr{R}_{K}[L], x_{\zeta} x_{\xi} x_{\eta} \in\left(x_{\alpha}\right) \cup\left(x_{\beta}\right)$. In fact,

$$
x_{\zeta} x_{\xi} x_{\eta}=x_{i \cup\{b\}} x_{\alpha} x_{\eta}=x_{\zeta} x_{\beta} x_{i \cup\{b\} \cup\left[c_{1}, c_{m}\right]} .
$$

First, we show, in general, that if $\gamma, \delta \in L$ with $\gamma \leq \delta$ and if $x_{\gamma} x_{\delta} \in\left(x_{\alpha}\right) \cap$ $\left(x_{\beta}\right)$, then $c_{1} \in \gamma$ and $c_{m} \notin \delta$. Let $x_{\gamma} x_{\delta}=x_{\alpha} x_{\alpha^{\prime}}=x_{\beta} x_{\beta^{\prime}}$ for some $\alpha^{\prime}, \beta^{\prime} \in L$. Since $a \in \beta$, we have $a \in \beta \vee \beta^{\prime}=\alpha \vee \alpha^{\prime}$. Thus, $a \in \alpha^{\prime}$. Hence, $c_{1} \in \alpha^{\prime}$. Then, $c_{1} \in \alpha \wedge \alpha^{\prime}=\gamma$. Since $b \notin \alpha$, we have $b \notin \alpha \wedge \alpha^{\prime}=\beta \wedge \beta^{\prime}$. Thus, $b \notin \beta^{\prime}$. Hence, $c_{m} \notin \beta^{\prime}$. Then, $c_{m} \notin \beta \vee \beta^{\prime}=\delta$.

Now, if $x_{\zeta} x_{\xi} x_{\eta}$ is not a generator of $\left(x_{\alpha}\right) \cap\left(x_{\beta}\right)$, then $x_{\gamma} x_{\delta} x_{\epsilon}$ for some $\gamma, \delta, \epsilon \in L$ with $x_{\gamma} x_{\delta} \in\left(x_{\alpha}\right) \cap\left(x_{\beta}\right)$ and $\gamma \leq \delta$. Since $c_{m} \in \delta$ (thus $c_{m} \notin \gamma$ ) and since $c_{m} \in \eta, c_{m}$ must belong to $\epsilon$. Hence, $c_{1} \in \epsilon$. Since $c_{1} \in \gamma$ (thus $\left.c_{1} \in \delta\right), c_{1}$ belongs to each of $\gamma, \delta$ and $\epsilon$. However, $c_{1} \notin \zeta$, a contradiction. Hence, $x_{\zeta} x_{\xi} x_{\eta}$ must be a generator of $\left(x_{\alpha}\right) \cap\left(x_{\beta}\right)$. Thus, $\mathscr{R}_{K}[L]$ is not strongly Koszul as required. 
Lemma 3.5. Every simple and connected (finite) poset $P$ possesses a saturated chain $c_{1}<c_{2}<\ldots<c_{m}(m \geq 2)$ together with $a, b \in P$ satisfying the above conditions (i)-(iv) in Lemma 3.4.

Proof. We denote minimal elements $w$ and $w^{\prime}$ in $P$ with $w \neq w^{\prime}$. If $P \backslash\{w\}$ is simple and connected, then we can find a required chain together with two elements in $P \backslash\{w\}$.

First, suppose that $P \backslash\{w\}$ is not connected. Let $Q_{1}, \ldots, Q_{s}(s \geq 2)$ denote the connected components of $P \backslash\{w\}$ with $w^{\prime} \in Q_{1}$. We choose a walk.

$$
w=z_{1} \rightarrow z_{2} \rightarrow \cdots \rightarrow z_{t-1} \rightarrow z_{t}=w^{\prime}
$$

with $z_{i} \neq z_{j}$ if $i \neq j$ combining $w$ with $w^{\prime}$ in $Q_{1} \cup\{w\}$. Here, $\rightarrow$ is the covering relation, i.e., for each $1 \leq i<t$, either $z_{i}$ covers $z_{i+1}$ or $z_{i}$ is covered by $z_{i+1}$. Let $2 \leq j<t$ denote the maximal integer for which $w \leq z_{j}$ and construct a saturated chain $w=c_{1}<c_{2}<\cdots<c_{m}=z_{j}$. Then, $x_{j+1}<z_{j}$ since $w \not \leq z_{j+1}$. Also, choose $y \in Q_{2}$ which covers $w$ in $P$. Then, the saturated chain $w=c_{1}<c_{2}<\cdots<c_{m}=z_{j}$ together with $a=y(>w)$ and $b=z_{j+1}\left(<z_{j}\right)$ satisfy the conditions in Lemma 3.4.

Second, suppose that $P \backslash\{w\}$ is not simple. Let $p_{1}, \ldots, p_{t}(t \geq 1)$ denote the elements such that each $p_{i}$ is comparable with an arbitrary element in $P \backslash\{w\}$ and asume that $p_{1}<\cdots<p_{t}$ in $P$. We choose $z \in P$ which covers $w$ in $P$. Then, $z>p_{t}$ since $P$ is simple. Also, choose a saturated chain $p_{t}=y_{1}<y_{2}<\cdots<y_{t}$ in $P$ such that $y_{t}$ is not comparable with $z$ in $P$. Let $1 \leq j<t$ denote the maximal integer for which $y_{j}<z$ and construct a saturated chain $y_{j}=c_{1}<c_{2}<\cdots<c_{m}=z$. Then, the saturated chain $y_{j}=c_{1}<c_{2}<\cdots<c_{m}=z$ together with $a=y_{j+1}\left(>y_{j}\right)$ and $b=w(<z)$ satisfy the conditions in Lemma 3.4.

\section{The classification of strongly Koszul algebras which are defined by bi- partite graphs}

Let $G$ be a graph on the vertex set $V=\{1, \ldots, n\}$. To $G$ we associate the subring $K[G]$ of the polynomial ring $K\left[x_{1}, \ldots, x_{n}\right]$ which is generated by all monomials $x_{i} x_{j}$ for which $(i, j)$ is an edge of $G$. We call the graph $G$ strongly Koszul, if $K[G]$ is strongly Koszul.

For example, if we let $G$ be the complete graph, then $K[G]$ is the second Veronese subring of $K\left[x_{1}, \ldots, x_{n}\right]$, and we know from 2.3 that $G$ is strongly Koszul. On the other hand, if we let $G$ be the complete graph without loops, that is, $G$ has all possible edges, except the loops $(i, i)$, then $K[G]$ is a second squarefree Veronese ring, and it follows from Example 1.6 (2), that $G$ is not strongly Koszul for $n \geq 5$. 
The question arises which graphs $G$ are strongly Koszul. We will restrict ourselves to consider bipartite graphs, because we do not know the general answer. Recall that a graph $G$ on a vertex set $V$ is called bipartite if the vertex set can be writen as a disjoint union $V=V_{1} \cup V_{2}$ such that all edges of $G$ are of the form $(i, j)$ with $i \in V_{1}$ nd $j \in V_{2}$. $G$ is called a complete $b i$ partite graph (on $\left(V_{1}, v_{2}\right)$ ), if all the edges $(i, j)$ with $i \in V_{2}$ belong to $G$. Notice that if $G$ is a complete bipartite graph on $\left(V_{1}, V_{2}\right)$, then $K[G]$ is the Segre product of the polynomial ring in the variables $x_{i}, i \in V_{1}$, and of the polynomial ring in the variables $x_{i}, i \in V_{2}$. Therefore, by 2.3, $G$ is strongly Koszul. We shall see that these are essentially the only strongly Koszul grphs.

We first show

Lemma 4.1. Every cycle of a strongly Koszul bipartite graph $G$ has all possible chords.

Proof. Notice that a cycle $C$ in a bipartite graph necessarily has an even number of vertices. Indeed, we may assume that $(1,2),(2,3), \ldots,(m-1, m)$ and $(m, 1)$ are the edges of the cycle. Then, if $1 \in V_{1}$, then all the "odd" vertices $2 i+1$ belong to $V_{1}$ and the "even" vertices to $V_{2}$. In particular, $m=2 k$ for some integer $k \geq 2$.

Now assume that some possible chord of $C$ does not belong to $G$. We may assume that this is cord $(1,2 i)$ for some $i$ with $1<i<k$. We consider the elements

$$
u=\prod_{j=1}^{i-1}\left(x_{2 j} x_{2 j+1}\right) \quad \text { and } \quad v=\prod_{j=i}^{k-1}\left(x_{2 j+1} x_{2 j+2}\right) .
$$

Then $\operatorname{deg} u=i-1, \operatorname{deg} v=k-i$ and $u v$ is the only element of $(u) \cap(v)$ of degree $k-1$. On the other hand, we see that the element $w=\prod_{i=1}^{k} x_{i}$ belongs to $(u) \cap(v)$. We have that $w$ is not a multiple of $u v$, because the remaining factor is just the missing chord. So $w$ is a generator of $(u) \cap(v)$ of degree $k$, contradicting 1.5.

Lemma 4.2. If $C_{1}$ and $C_{2}$ are cycles of a (not necessarily bipartite) graph and if there exist at least two common vertices of $C_{1}$ and $C_{2}$, then for any vertex $i$ of $C_{1}$ and for any vertex $j$ of $C_{2}$ we can find a cycle $C$ which contains both $i$ and $j$.

Proof. We may assume that $i$ does not belong to $C_{2}$ and that $j$ does not belong to $C_{1}$. Let $\alpha$ and $\beta$ denote two common vertices of $C_{1}$ and $C_{2}$ such that $i$ belongs to an $\operatorname{arc} \Gamma$ of $C_{1}$ between $\alpha$ and $\beta$, and that no vertex $(\neq \alpha, \beta)$ of $\Gamma$ belongs to $C_{2}$. We then choose an arc $\Gamma^{\prime}$ of $C_{2}$ between $\alpha$ and $\beta$ such that $j$ belongs to $\Gamma^{\prime}$. Now, $\Gamma \cup \Gamma^{\prime}$ is a cycle which contains both $i$ and $j$, as required. 
Corollary 4.3. Let $C_{1}$ and $C_{2}$ be two cycles of a strongly Kosul bipartite graph $G$ which have at least one common edge. Then there exists a complete bipartite subgraph of $G$ which contains both $C_{1}$ and $C_{2}$.

Proof. Let $V_{1}$ and $V_{2}$ be the partition of the vertex set $V$ of $G$. Let $i \in V_{1}$ and $j \in V_{2}$ and suppose that both vertices belong to $C_{1} \cup C_{2}$. We will show that $(i, j)$ is an edge of $G$. If both the vertices belong to either $C_{1}$ or $C_{2}$, then the assertion follows from 4.1. Now suppose that $i$ is a vertex of, say, $C_{1}$ and $j$ a vertex of $C_{2}$. Since $C_{1}$ and $C_{2}$ have an edge in common, by 4.2 we can find a cycle $C$ (which is a subgraph of $C_{1} \cup C_{2}$ ) with the vertices $i$ and $j$. Now we apply again 4.1 in order to conclude that $(i, j)$ is an edge of $G$.

One more ingredient is needed in order to prove the main result of this section. Let $G$ be a bipartite graph. We want to describe the relations of $K[G]$. Consider the polynomial ring $P$ defined over $K$ in the indeterminates $y_{i j}$ for all $(i, j)$ which are edges of $G$. For convenience $Y_{i j}$ and $y_{j i}$ will be identified. Let $I$ be the kernel of the $K$-algebra homomorphism which sends $y_{i j}$ to $x_{i} x_{j}$. To each even closed walk $\Gamma$ of $G$ with edges $\left(i_{1}, i_{2}\right),\left(i_{2}, i_{3}\right), \ldots,\left(i_{2 k-1}, i_{2 k}\right),\left(i_{2 k}, i_{1}\right)$ we assign the relation

$$
f_{\Gamma}=\prod_{j=1}^{k} y_{i_{2 j-1,}, i_{2 j}}-\prod_{j=1}^{k} y_{i_{2 j}, i_{2 j+1}}
$$

with $i_{2 k+1}=i_{1}$.

Lemma 4.4. The relation ideal I of $K[G]$ is generated by all the relations $f_{C}$, where $C$ is a cycle of $G$.

Proof. It follows from, e.g., [12, Proof of Theorem 9.1] that $I$ is generated by all the relations $f_{\Gamma}$, where $\Gamma$ is an even closed walk of $G$. Let $\Gamma$ be an even closed walk with edges $\left(i_{1}, i_{2}\right),\left(i_{2}, i_{3}\right), \ldots,\left(i_{2 k-1}, i_{2 k}\right),\left(i_{2 k}, i_{1}\right)$ and suppose that $\Gamma$ is not a cycle. We may assume that $i_{2 p+1}=i_{1}$ for some $1 \leq p<k$. Let $\Gamma_{1}$ denote the even closed subwalk with edges $\left(i_{1}, i_{2}\right),\left(i_{2}, i_{3}\right), \ldots,\left(i_{2 p-1}, i_{2 p}\right)$, $\left(i_{2 p}, i_{1}\right)$ and $\Gamma_{2}$ the even closed subwalk with edges $\left(i_{1}, i_{2 p+2}\right),\left(i_{2 p+2}, i_{2 p+3}\right), \ldots$, $\left(i_{2 k-1}, i_{2 k}\right),\left(i_{2 k}, i_{1}\right)$. Then, we have 


$$
\begin{aligned}
f_{\Gamma}= & \prod_{j=1}^{k} y_{i_{2 j-1}, i_{2 j}}-\prod_{j=1}^{k} y_{i_{2 j}, i_{2 j+1}} \\
= & \prod_{j=1}^{k} y_{i_{2 j-1}, i_{2 j}}-\left(\prod_{j=1}^{p} y_{i_{2 j-1}, i_{2 j}}\right)\left(\prod_{j=p+1}^{k} y_{i_{2_{j}, i_{2 j+1}}}\right) \\
& +\left(\prod_{j=1}^{p} y_{i_{2 j-1}, i_{2 j}}\right)\left(\prod_{j=p+1}^{k} y_{i_{2 j}, i_{2 j+1}}\right)-\prod_{j=1}^{k} y_{i_{2 j}, i_{2 j+1}} \\
= & \left(\prod_{j=1}^{p} y_{i_{2 j-1}, i_{2 j}}\right)\left(\prod_{j=p+1}^{k} y_{i_{2 j-1}, i_{2 j}}-\prod_{j=p+1}^{k} y_{i_{2 j}, i_{2 j+1}}\right) \\
& +\left(\prod_{j=1}^{p} y_{i_{2 j-1}, i_{2 j}}-\prod_{j=1}^{p} y_{i_{2 j}, i_{2 j+1}}\right)\left(\prod_{j=p+1}^{k} y_{i_{2 j}, i_{2 j+1}}\right) \\
= & \left(\prod_{j=1}^{p} y_{i_{2 j-1}, i_{2 j}}\right) f_{\Gamma_{2}}+f_{\Gamma_{1}}\left(\prod_{j=p+1}^{k} y_{i_{2 j}, i_{2 j+1}}\right) .
\end{aligned}
$$

Hence, $f_{\Gamma}$ belongs to the ideal generated by all the relations $f_{\Gamma_{1}}$ and $f_{\Gamma_{2}}$. Thus, repeated applications of the technique guarantee that $f_{\Gamma}$ belongs to the ideal generated by all the relations $f_{C}$, where $C$ is a cycle of $G$, as desired.

Now we are ready for the proof of

TheOREM 4.5. Let $G$ be a strongly Koszul bipartite graph, and let $G_{1}, \ldots, G_{p}$ be the maximal complete bipartite subgraphs of $G$. Then $K[G] \cong K\left[G_{1}\right] \otimes \ldots \otimes$ $K\left[G_{p}\right]$. In particular, since each $K\left[G_{i}\right]$ is the Segre product of two polynomials rings, the semigroup defined by $G$ is trivial.

Proof. Thanks to 4.3 , if $e=(i, j)$ is an edge of $G_{r}$ and $e^{\prime}=\left(i^{\prime}, j^{\prime}\right)$ is an ede of $G_{s}$ with $r \neq s$, then no cycle contains both $e$ and $e^{\prime}$. Since by 4.4 , the cycle relations generate the defining ideal of $K[G]$, we see that there exist no generating relations involving variables corresponding to $G_{r}$ and $G_{s}$. This implies that $K[G] \cong K\left[G_{1}\right] \otimes \ldots \otimes K\left[G_{p}\right]$, as desired.

\section{REFERENCES}

1, J. Backelin, On the rates of growth of the homologies of Veronese subrings, in: Algebra, Algebraic Topology and Their Interactions, J.-E. Roos, Ed., Lecture Notes in Math. 1183 (1986), 79-100.

2. J. Backelin and R. Fröberg, Koszul algebras, Veronese subrings, and rings with linear resolutions, Rev. Roum. Math. Pures Appl. 30 (1985), 549-565.

3. G. Birkhoff, Lattice theory, 3rd. ed., Amer. Math. Soc. Colloq. Publ. No. 25, 1967.

4. W. Bruns, J. Herzog and U. Vetter, Syzygies and Walks, in: ICTP Proceedings Commutative Algebra, A. Simis, N. V. Trung and G. Valla, Eds., World Scientific 1994, 36-57. 
5. R. Fröberg, Determination of a Class of Poincaré series, Math. Scand. 37 (1975), 29-39.

6. T. Hibi, Distributive lattices, affine semigroup rings, and algebras with straightening laws, in: Commutative Algebra and Combinatorics, M. Nagata and H. Matsumura, Eds., Adv. Stud. Pure Math. 11, 1987, 93-103.

7. T. Hibi, Algebraic Combinatorics on Convex Polytopes, Carslaw Publications, Glebe, N.S.W., Australia, 1992.

8. O. A. Laudal and A. Sletsjøe, Betti numbers of monoid algebras: Applications to 2-dimensional torus embeddings, Math. Scand. 56 (1985), 145-162.

9. J. Herzog, V. Reiner and V. Welker, The Koszul property in affine semigroup rings, Pac. J. Math. 186 (1998), 39-65.

10. E. De Negri, Toric rings generated by stable sets of monomials, Math. Nachr. 203 (1999), 3145.

11. I. Peeva, V. Reiner and B. Sturmfels, How to shell a monoid, Math. Ann. 310 (1998), 379194.

12. B. Sturmfels, Gröbner Bases and Convex Polytopes, Univ. Lecture Ser. 8 (1995).

FB6 MATHEMATIK UND INFORMATIK UNIVERSIÄT GH-ESSEN

45117 ESSEM

GERMANY

mat300@@une-essen.de
DEPARTMENT OF MATHEMATICS GRADUATE SCHOOL OF SCIENCE OSAKA UNIVERSITY TOYONAKA, OSAKA 560, JAPAN

E-mail:hibi@@math.sci.osaka-uc.jp

DEPARTIMENTO DI MATEMATICA

UNIVERSITA DI MESSINA

CONTRADA PAPARDO SALITA SPERONE 3

98166 SANT'AGATA, MESSINA, ITALIA

E-mail: grest@@imeuniv.unime.it 